\title{
Hopping from Chebyshev polynomials to permutation statistics
}

\author{
Jordan O. Tirrell \\ Department of Mathematics and Statistics \\ Mount Holyoke College \\ South Hadley, MA, U.S.A. \\ jtirrell@mtholyoke.edu \\ Yan Zhuang \\ Department of Mathematics and Computer Science \\ Davidson College \\ Davidson, NC, U.S.A. \\ yazhuang@davidson.edu
}

Submitted: Dec 27, 2018; Accepted: Jul 17, 2019; Published: Aug 16, 2019

(c) The authors. Released under the CC BY license (International 4.0).

\begin{abstract}
We prove various formulas which express exponential generating functions counting permutations by the peak number, valley number, double ascent number, and double descent number statistics in terms of the exponential generating function for Chebyshev polynomials, as well as cyclic analogues of these formulas for derangements. We give several applications of these results, including formulas for the $(-1)$-evaluation of some of these distributions. Our proofs are combinatorial and involve the use of monomino-domino tilings, the modified Foata-Strehl action (a.k.a. valley-hopping), and a cyclic analogue of this action due to Sun and Wang.
\end{abstract}

Mathematics Subject Classifications: 05A15, 05A05, 33C45

\section{Introduction}

Let $\pi=\pi_{1} \pi_{2} \cdots \pi_{n}$ be a permutation (written in one-line notation) in $\mathfrak{S}_{n}$, the set of permutations of $[n]=\{1,2, \ldots, n\}$. We say that $\pi_{i}$ (where $i \in[n-1]$ ) is a descent if $\pi_{i}>\pi_{i+1}$, and that $\pi_{i}$ (where $2 \leqslant i \leqslant n-1$ ) is a peak of $\pi$ if $\pi_{i-1}<\pi_{i}>\pi_{i+1}$. Define $\operatorname{des}(\pi)$ to be the number of descents of $\pi$ and $\mathrm{pk}(\pi)$ to be the number of peaks of $\pi$. The descent number des and peak number pk are classical permutation statistics whose study dates back to MacMahon [11] and to David and Barton [7], respectively. 
The $n$th Eulerian polynomial ${ }^{1}$

$$
A_{n}(t):=\sum_{\pi \in \mathfrak{S}_{n}} t^{\operatorname{des}(\pi)}
$$

encodes the distribution of the descent number des over $\mathfrak{S}_{n}$, and the $n$th peak polynomial

$$
P_{n}^{\mathrm{pk}}(t):=\sum_{\pi \in \mathfrak{S}_{n}} t^{\mathrm{pk}(\pi)}
$$

is the analogous polynomial for the peak number pk.

It is well-known [9, Théorème 5.6] that the (-1)-evaluation of the Eulerian distribution is given by the formula

$$
A_{n}(-1)= \begin{cases}(-1)^{(n-1) / 2} E_{n}, & \text { if } n \text { is odd } \\ 0, & \text { if } n \text { is even }\end{cases}
$$

where $E_{n}$ is the $n$th Euler number defined by

$$
\sum_{n=0}^{\infty} E_{n} \frac{x^{n}}{n !}=\sec (x)+\tan (x) .
$$

(The Euler numbers $E_{n}$ for odd $n$ are called tangent numbers, and those for even $n$ are called secant numbers.) No combinatorial formula for $P_{n}^{\mathrm{pk}}(-1)$ is known, although this sequence does appear on the OEIS [18, A006673]. The first several terms of this sequence are given in the following table:

\begin{tabular}{c|cccccccccc}
$n$ & 1 & 2 & 3 & 4 & 5 & 6 & 7 & 8 & 9 & 10 \\
\hline$P_{n}^{\mathrm{pk}}(-1)$ & 1 & 2 & 2 & -8 & -56 & -112 & 848 & 9088 & 25216 & -310528
\end{tabular}

We note that the apparent 6 -periodicity of the sequence of signs breaks at $n=42$. Very recently, Troyka [22] argued that there is no $k$ for which the sequence of signs of the $P_{n}^{\mathrm{pk}}(-1)$ is $k$-periodic, which suggests that there is unlikely to be a nice combinatorial interpretation for the $P_{n}^{\mathrm{pk}}(-1)$.

The exponential generating functions for $A_{n}(t)$ and $P_{n}^{\mathrm{pk}}(t)$ have the following well-known expressions: ${ }^{2}$

$$
\begin{gathered}
A(t ; x):=\sum_{n=1}^{\infty} A_{n}(t) \frac{x^{n}}{n !}=\frac{e^{(1-t) x}-1}{1-t e^{(1-t) x}} ; \\
P^{\mathrm{pk}}(t ; x):=\sum_{n=1}^{\infty} P_{n}^{\mathrm{pk}}(t) \frac{x^{n}}{n !}=\frac{1}{\sqrt{1-t} \operatorname{coth}(x \sqrt{1-t})-1} .
\end{gathered}
$$

\footnotetext{
${ }^{1}$ We note that many works instead define the $n$th Eulerian polynomial to be $\sum_{\pi \in \mathfrak{S}_{n}} t^{\operatorname{des}(\pi)+1}$.

${ }^{2}$ These exponential generating functions are usually given a constant term of 1 in the literature, but it is more convenient to define these without the constant term in this work.
} 
The work in this paper was originally inspired by the curious observation that $A(-1 ; x)$ and $P^{\mathrm{pk}}(-1 ; x)$ can be expressed as the logarithmic derivative of the exponential generating function of some non-negative integer sequence. For the Eulerian polynomials, this sequence $\left\{f_{n}\right\}_{n \geqslant 0}$ is simply $f_{n}:=(n+1) \bmod 2$, i.e., the sequence $1,0,1,0, \ldots$, whose exponential generating function is given by

$$
F(x):=1+\frac{x^{2}}{2 !}+\frac{x^{4}}{4 !}+\cdots=\cosh (x) .
$$

For the peak polynomials, this sequence is the sequence of Pell numbers, which has been widely studied in combinatorics and number theory. The Pell numbers $\left\{g_{n}\right\}_{n \geqslant 0}$ are defined by the recursive formula $g_{n}:=2 g_{n-1}+g_{n-2}$ for $n \geqslant 2$ with initial values $g_{0}=1$ and $g_{1}=0$. The first several terms of this sequence are below:

\begin{tabular}{c|ccccccccccccc}
$n$ & 0 & 1 & 2 & 3 & 4 & 5 & 6 & 7 & 8 & 9 & 10 & 11 & 12 \\
\hline$g_{n}$ & 1 & 0 & 1 & 2 & 5 & 12 & 29 & 70 & 169 & 408 & 985 & 2378 & 5741
\end{tabular}

Note that the indexing here is slightly different from the usual indexing of the Pell numbers (see OEIS [18, A000129]). The exponential generating function of $\left\{g_{n}\right\}_{n \geqslant 0}$ is given by

$$
G(x):=\sum_{n=0}^{\infty} g_{n} \frac{x^{n}}{n !}=\frac{1}{2} e^{x}(2 \cosh (x \sqrt{2})-\sqrt{2} \sinh (x \sqrt{2})) .
$$

Theorem 1. The exponential generating functions for the Eulerian and peak polynomials evaluated at $t=-1$ can be expressed as the logarithmic derivative of $F(x)$ and $G(x)$, respectively. That is:

(a) $A(-1 ; x)=\frac{\frac{d}{d x} F(x)}{F(x)}$

(b) $P^{\mathrm{pk}}(-1 ; x)=\frac{\frac{d}{d x} G(x)}{G(x)}$

While Theorem 1 can be proven directly by algebraically manipulating the generating function formulas for $A(t ; x), P^{\mathrm{pk}}(t ; x), F(x)$, and $G(x)$, one of our goals in this paper is to present a combinatorially-flavored proof. In Section 2, we define several other relevant permutation statistics and introduce a key ingredient of our proof: the modified Foata-Strehl group action (a.k.a. valley-hopping). In Section 3, we define a two-parameter variant of the Chebyshev polynomials of the second kind which specialize to both the numbers $f_{n}$ and the Pell numbers $g_{n}$. Like the ordinary Chebyshev polynomials of the second kind, our bivariate Chebyshev polynomials have as a combinatorial model monomino-domino tilings of a rectangle, but with slightly different weights. We present a formula (Theorem 3) involving these Chebyshev polynomials for the joint distribution of two statistics: the peak number, and the total number of double ascents and double descents. We give a combinatorial proof of Theorem 3 which involves tilings and valleyhopping, and a special case of this result implies Theorem 1 (b). We transform Theorem 3 
into similar results for other permutation statistics, which we then use to prove Theorem 1 (a) and to prove that the (-1)-evaluation of double descent distributions yields the tangent numbers.

In Section 4, we turn our attention to counting derangements by cyclic analogues of the permutation statistics studied in Sections 2-3. Using a variant of valley-hopping due to Sun and Wang [21] for derangements, we prove a cyclic analogue of Theorem 3 and use it to derive formulas relating exponential generating functions counting derangements by cyclic statistics with the exponential generating function for our Chebyshev polynomials. We use this to prove a result similar to Theorem 1 for the excedance and cyclic peak distributions over derangements, and to prove that the (-1)-evaluation of cyclic double descent distributions over derangements yields the secant numbers.

\section{Permutation statistics and valley-hopping}

Given a permutation $\pi=\pi_{1} \pi_{2} \cdots \pi_{n}$ in $\mathfrak{S}_{n}$, we say that $\pi_{i}$ (where $i \in[n]$ ) is:

- a valley if $\pi_{i-1}>\pi_{i}<\pi_{i+1}$;

- a double ascent if $\pi_{i-1}<\pi_{i}<\pi_{i+1}$;

- a double descent if $\pi_{i-1}>\pi_{i}>\pi_{i+1}$;

where we are using the convention $\pi_{0}=\pi_{n+1}=\infty$. $^{3}$ Thus, every letter of a permutation is either a peak, valley, double ascent, or double descent. Define $\operatorname{val}(\pi), \operatorname{dasc}(\pi)$, and $\operatorname{ddes}(\pi)$ to be the number of valleys, double ascents, and double descents of $\pi$, respectively. We also define $\operatorname{dbl}(\pi):=\operatorname{dasc}(\pi)+\operatorname{ddes}(\pi)$ to be the total number of double ascents and double descents of $\pi$.

For a list of statistics $\mathrm{st}_{1}, \mathrm{st}_{2}, \ldots, \mathrm{st}_{m}$ and corresponding variables $t_{1}, t_{2}, \ldots, t_{m}$, we define the polynomials $\left\{P_{n}^{\left(\mathrm{st}_{1}, \mathrm{st}_{2}, \ldots \mathrm{st}_{m}\right)}\left(t_{1}, t_{2}, \ldots, t_{m}\right)\right\}_{n \geqslant 0}$ by

$$
P_{n}^{\left(\mathrm{st}_{1}, \mathrm{st}_{2}, \ldots \mathrm{st}_{m}\right)}\left(t_{1}, t_{2}, \ldots, t_{m}\right):=\sum_{\pi \in \mathfrak{S}_{n}} t_{1}^{\mathrm{st}_{1}(\pi)} t_{2}^{\mathrm{st}_{2}(\pi)} \cdots t_{m}^{\mathrm{st}_{m}(\pi)} .
$$

and we let

$$
P^{\left(\mathrm{st}_{1}, \mathrm{st}_{2}, \ldots \mathrm{st}_{m}\right)}\left(t_{1}, t_{2}, \ldots, t_{m} ; x\right):=\sum_{n=1}^{\infty} P_{n}^{\left(\mathrm{st}_{1}, \mathrm{st}_{2}, \ldots \mathrm{st}_{m}\right)}\left(t_{1}, t_{2}, \ldots, t_{m}\right) \frac{x^{n}}{n !}
$$

be their exponential generating function. ${ }^{4}$ For example, we have

$$
P_{n}^{(\mathrm{pk}, \mathrm{dbl})}(s, t)=\sum_{\pi \in \mathfrak{S}_{n}} s^{\mathrm{pk}(\pi)} t^{\mathrm{dbl}(\pi)}
$$

\footnotetext{
${ }^{3}$ We note that many works on permutation enumeration do not use these conventions, and simply restrict the possible positions of valleys, double ascents, and double descents to the interval from 2 to $n-1$. What we call "valleys" are sometimes called "left-right valleys" or "exterior valleys", what we call "double ascents" are sometimes called "right double ascents", and what we call "double descents" are sometimes called "left double descents". (See, e.g., [24].)

${ }^{4}$ In the case where we have a single statistic st, we write these simply as $P_{n}^{\text {st }}(t)$ and $P^{\text {st }}(t ; x)$.
} 
and

$$
P^{(\mathrm{pk}, \mathrm{dbl})}(s, t ; x)=\sum_{n=1}^{\infty} P_{n}^{(\mathrm{pk}, \mathrm{dbl})}(s, t) \frac{x^{n}}{n !} ;
$$

we will consider these on the way to proving Theorem 1. Our proof will make use of a bijection based on a group action on $\mathfrak{S}_{n}$ induced by involutions which toggle between double ascents and double descents; we will spend the remainder of this section defining this action and the associated bijection.

For $\pi \in \mathfrak{S}_{n}$, fix $k \in[n]$. We may write $\pi=w_{1} w_{2} k w_{4} w_{5}$ where $w_{2}$ is the maximal consecutive subword immediately to the left of $k$ whose letters are all smaller than $k$, and $w_{4}$ is the maximal consecutive subword immediately to the right of $k$ whose letters are all smaller than $k$. For example, if $\pi=467125839$ and $k=5$, then $\pi$ is the concatenation of $w_{1}=467, w_{2}=12, k=5$, the empty word $w_{4}$, and $w_{5}=839$.

Define $\varphi_{k}: \mathfrak{S}_{n} \rightarrow \mathfrak{S}_{n}$ by

$$
\varphi_{k}(\pi)= \begin{cases}w_{1} w_{4} k w_{2} w_{5}, & \text { if } k \text { is a double ascent or double descent of } \pi \\ \pi, & \text { if } k \text { is a peak or valley of } \pi\end{cases}
$$

Equivalently, $\varphi_{k}(\pi)=w_{1} w_{4} k w_{2} w_{5}$ if exactly one of $w_{2}$ and $w_{4}$ is nonempty, and $\varphi_{k}(\pi)=\pi$ otherwise. For any subset $S \subseteq[n]$, we define $\varphi_{S}: \mathfrak{S}_{n} \rightarrow \mathfrak{S}_{n}$ by $\varphi_{S}=\prod_{k \in S} \varphi_{k}$. It is easy to see that $\varphi_{S}$ is an involution, and that for all $S, T \subseteq[n]$, the involutions $\varphi_{S}$ and $\varphi_{T}$ commute with each other. Hence the involutions $\left\{\varphi_{S}\right\}_{S \subseteq[n]}$ define a $\mathbb{Z}_{2}^{n}$-action on $\mathfrak{S}_{n}$ which is often called the modified Foata-Strehl action or valley-hopping. This action is based on a classical group action of Foata and Strehl [10], was introduced by Shapiro, Woan, and Getu [15], and was later rediscovered by Brändén [4].

Let $\widetilde{\mathfrak{S}}_{n}$ denote the set of permutations of $[n]$ with no double ascents and where each double descent is assigned one of two colors: red or blue. ${ }^{5}$ Then valley-hopping induces a map $\Phi$ from $\widetilde{\mathfrak{S}}_{n}$ to $\mathfrak{S}_{n}$ defined in the following way. Given a permutation $\pi$ in $\widetilde{\mathfrak{S}}_{n}$, let $R(\pi)$ be the set of red double descents in $\pi$ and let $\bar{\pi}$ be the corresponding permutation of $\pi$ in $\mathfrak{S}_{n}$, that is, the permutation obtained by forgetting the colors on the double descents. Then let $\Phi(\pi)=\varphi_{R(\pi)}(\bar{\pi})$. For example, if $\pi=726539841$, then $\Phi(\pi)=265379418$. (See Figure 1.)

Lemma 2. The map $\Phi: \widetilde{\mathfrak{S}}_{n} \rightarrow \mathfrak{S}_{n}$ is a (pk, dbl)-preserving bijection.

Proof. The inverse $\Phi^{-1}$ of the map $\Phi$ can be described in the following way. Let Dasc $(\pi)$ be the set of double ascents of $\pi$ and $\operatorname{Ddes}(\pi)$ the set of double descents of $\pi$. If $S \subseteq \operatorname{Ddes}(\pi)$ and if $\pi$ has no double ascents, then let $\pi^{S}$ be the permutation in $\widetilde{\mathfrak{S}}_{n}$ obtained by coloring the double descents in $S$ blue and all other double descents red. Given a permutation $\pi$ in $\mathfrak{S}_{n}$, let $\Phi^{-1}(\pi)=\left(\varphi_{\operatorname{Dasc}(\pi)}(\pi)\right)^{\operatorname{Ddes}(\pi)}$. Then $\Phi$ is a bijection between $\widetilde{\mathfrak{S}}_{n}$ and $\mathfrak{S}_{n}$. The claim that $\Phi$ preserves the pk and $\mathrm{dbl}$ statistics follows from the easy fact that valley-hopping preserves these statistics as well.

\footnotetext{
${ }^{5}$ To be more precise, Davidson College red or Mount Holyoke College blue.
} 

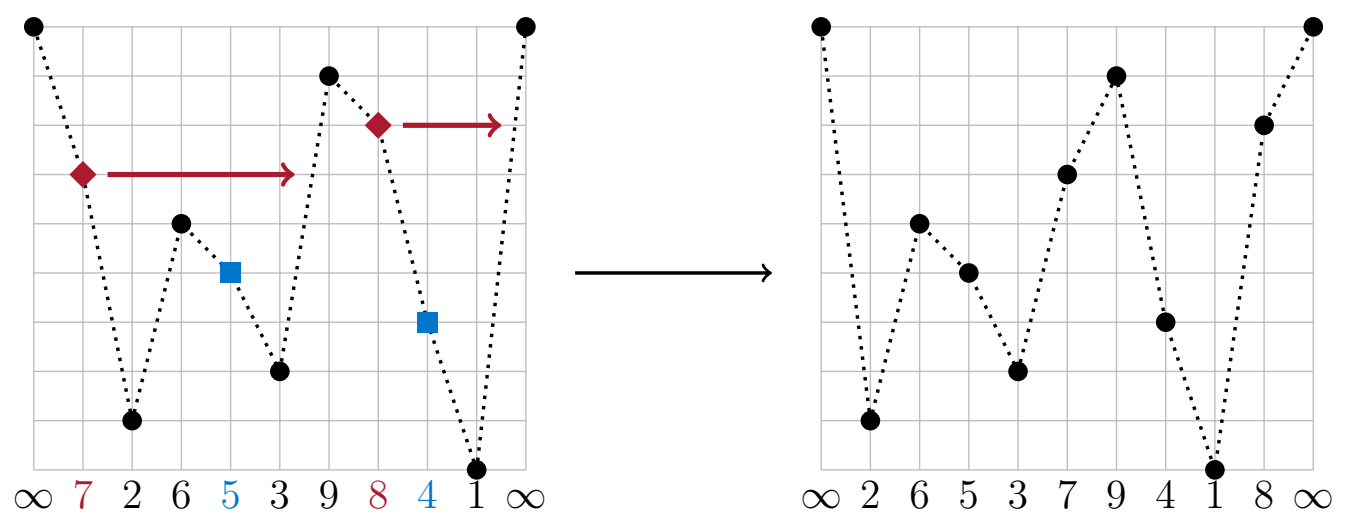

Figure 1: Valley-hopping induces a bijection between $\widetilde{\mathfrak{S}}_{n}$ and $\mathfrak{S}_{n}$.

\section{Hopping from Chebyshev polynomials to permutation statis- tics}

The Chebyshev polynomials of the second kind $\left\{U_{n}(t)\right\}_{n \geqslant 0}$ are defined by the recurrence $U_{n}(t):=2 t U_{n-1}(t)-U_{n-2}(t)$ for $n \geqslant 2$ with initial values $U_{0}(t)=1$ and $U_{1}(t)=2 t$. These polynomials are an important sequence of orthogonal polynomials arising in many branches of mathematics; see $[1,12,13]$ for several notable references.

It will be more convenient for us to use a two-parameter variant $\left\{U_{n}(s, t)\right\}_{n \geqslant 0}$ of the Chebyshev polynomials of the second kind. We define $U_{n}(s, t)$ by the recurrence

$$
U_{n}(s, t)=2 t U_{n-1}(s, t)-s U_{n-2}(s, t)
$$

for $n \geqslant 2$ with initial values $U_{0}(s, t)=1$ and $U_{1}(s, t)=2 t$. The first several of these bivariate Chebyshev polynomials are given in the following table.

\begin{tabular}{c|c}
$n$ & $U_{n}(s, t)$ \\
\hline 0 & 1 \\
1 & $2 t$ \\
2 & $4 t^{2}-s$ \\
3 & $8 t^{3}-4 s t$
\end{tabular}

\begin{tabular}{c|c}
$n$ & $U_{n}(s, t)$ \\
\hline 4 & $16 t^{4}-12 s t^{2}+s^{2}$ \\
5 & $32 t^{5}-32 s t^{3}+6 s^{2} t$ \\
6 & $64 t^{6}-80 s t^{4}+24 s^{2} t^{2}-s^{3}$ \\
7 & $128 t^{7}-192 s t^{5}+80 s^{2} t^{3}-8 s^{3} t$
\end{tabular}

Note that these polynomials are related to the usual Chebyshev polynomials of the second kind by the formulas $U_{n}(t)=U_{n}(1, t)$ and $U_{n}(s, t)=U_{n}\left(s^{-1 / 2} t\right) s^{n / 2}$. The numbers $f_{n}=(n+1) \bmod 2$ and the Pell numbers $g_{n}$ are specializations of the $U_{n}(s, t)$, as $f_{n}=$ $U_{n-2}(-1,0)$ and $g_{n}=U_{n-2}(-1,1)$ for all $n \geqslant 2$. From the recurrence $(2)$, it is not hard to see that the ordinary generating function for our $U_{n}(s, t)$ is given by the formula

$$
\sum_{n=0}^{\infty} U_{n}(s, t) x^{n}=\frac{1}{1-2 t x+s x^{2}},
$$

and that $U_{n}(s, t)$ counts tilings of a $1 \times n$ rectangle with two types of monominoes, each weighted $t$, and one type of domino, each weighted $-s$. (The ordinary Chebyshev 
polynomials of the second kind count the same tilings but with dominoes weighted -1 ; see [3] for an accessible reference.)

An expression for the exponential generating function of the $U_{n}(t)$ is known (see [12, p. 301]) and together with the formula $U_{n}(s, t)=U_{n}\left(s^{-1 / 2} t\right) s^{n / 2}$, we obtain

$$
\sum_{n=0}^{\infty} U_{n}(s, t) \frac{x^{n+1}}{(n+1) !}=e^{x t} \frac{\sinh \left(x \sqrt{t^{2}-s}\right)}{\sqrt{t^{2}-s}} .
$$

We will find it more convenient to work with the exponential generating function

$$
V(s, t ; x):=\sum_{n=0}^{\infty} U_{n}(s, t) \frac{x^{n+2}}{(n+2) !}=\frac{x^{2}}{2 !}+2 t \frac{x^{3}}{3 !}+\left(4 t^{2}-s\right) \frac{x^{4}}{4 !}+\cdots .
$$

Note that

$$
F(x)=\sum_{n=0}^{\infty} f_{n} \frac{x^{n}}{n !}=1+V(-1,0 ; x) \quad \text { and } \quad G(x)=\sum_{n=0}^{\infty} g_{n} \frac{x^{n}}{n !}=1+V(-1,1 ; x) .
$$

It follows from $(3)$ that $V(s, t ; x)$ has the closed-form expression

$$
V(s, t ; x)=\frac{1}{s}\left(1-\cosh \left(x \sqrt{t^{2}-s}\right) e^{t x}+\frac{t e^{t x} \sinh \left(x \sqrt{t^{2}-s}\right)}{\sqrt{t^{2}-s}}\right) .
$$

\subsection{A Chebyshev formula for the bidistribution (pk, dbl)}

We now present our main theorem from this section.

Theorem 3. $P^{(\mathrm{pk}, \mathrm{dbl})}(s, t ; x)=\frac{\frac{\partial}{\partial x} V(s, t ; x)}{1-s V(s, t ; x)}$

Setting $s=-1$ and $t=1$ in Theorem 3 yields Theorem 1 (b). Observe that the numerator in Theorem 3 appears in Equation (3), and using Equation (4) for the denominator we can obtain the expression

$$
P^{(\mathrm{pk}, \mathrm{dbl})}(s, t ; x)=\frac{1}{\sqrt{t^{2}-s} \operatorname{coth}\left(x \sqrt{t^{2}-s}\right)-t} .
$$

Proof. From the combinatorial interpretation of multiplication of exponential generating functions (see, e.g., [20, Proposition 5.1.3]), it suffices to show that

$$
P_{n}^{(\mathrm{pk}, \mathrm{dbl})}(s, t)=\sum_{k=0}^{\lfloor(n-1) / 2\rfloor} s^{k} \sum_{B} U_{\left|B_{0}\right|-1}(s, t) U_{\left|B_{1}\right|-2}(s, t) \cdots U_{\left|B_{k}\right|-2}(s, t)
$$

where the second sum is over all ordered set partitions $B$ of $[n]$ into blocks $B_{0}, B_{1}, \ldots, B_{k}$ such that every block other than $B_{0}$ has size at least 2. Thus, the right-hand side of Equation (5) counts these set partitions together with: 


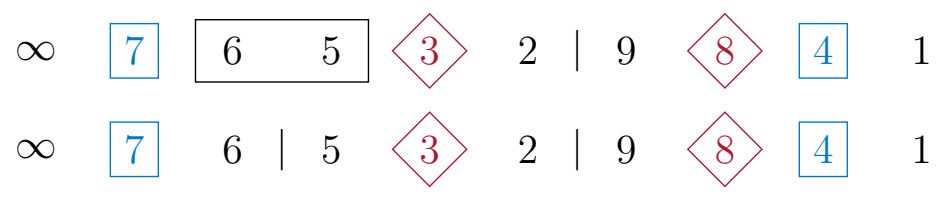

Figure 2: Example of the sign-reversing involution.

- a tiling of an $1 \times\left(\left|B_{0}\right|-1\right)$ rectangle with two types of monominoes (colored red and blue), each weighted $t$, and one type of domino, each weighted $-s$;

- for each $1 \leqslant i \leqslant k$, a tiling of an $1 \times\left(\left|B_{i}\right|-2\right)$ rectangle with the same types of shapes and weights as above;

and each block (other than $B_{0}$ ) is given an additional weight of $s$. We place an $\infty$ in the first block, write out each block in decreasing order, and separate adjacent blocks with a bar, as in

$$
\infty>\pi_{1}>\pi_{2}>\cdots>\pi_{\left|B_{0}\right|}\left|\pi_{\left|B_{0}\right|+1}>\cdots>\pi_{\left|B_{0}\right|+\left|B_{1}\right|}\right| \cdots \mid \pi_{n-\left|B_{k}\right|+1}>\cdots>\pi_{n} .
$$

Here, we consider the tiling on each block as being a tiling on all but the first and last elements of the block. Now we define a sign-reversing involution on these objects in the following way: Find the first pair of elements $\left(\pi_{i}, \pi_{i+1}\right)$ where there is a domino, or where $\pi_{i}$ and $\pi_{i+1}$ are in separate blocks and $\pi_{i}>\pi_{i+1}$. If $\left(\pi_{i}, \pi_{i+1}\right)$ is covered by a domino, then we remove the domino and insert a new bar in between $\pi_{i}$ and $\pi_{i+1}$, thus splitting their block into two blocks. If $\pi_{i}$ and $\pi_{i+1}$ are in separate blocks and $\pi_{i}>\pi_{i+1}$, then we merge the two blocks and cover $\left(\pi_{i}, \pi_{i+1}\right)$ with a domino. (See Figure 2.) This involution swaps a domino (weighted $-s$ ) with an additional block (weighted $s$ ), and after cancellation we are left with those objects with no dominoes and such that $\pi_{i}<\pi_{i+1}$ whenever $\pi_{i}$ and $\pi_{i+1}$ are in separate blocks.

If we treat any one of these remaining objects $\pi=\pi_{1} \pi_{2} \cdots \pi_{n}$ as a permutation, we see that $\pi$ has no double ascents and has each double descent colored either red or blue (depending on the color of the corresponding monomino). Hence, $\pi$ belongs to $\widetilde{\mathfrak{S}}_{n}$ and contributes a weight of $s^{\mathrm{pk}(\pi)} t^{\mathrm{dbl}(\pi)}$ to the right-hand side of Equation (5). The result then follows from applying the (pk, dbl)-preserving bijection $\Phi$.

\subsection{A Chebyshev formula for the quadruple distribution (pk, val, dasc, ddes)}

We shall now derive from Theorem 3 an analogous result for the joint distribution of the four statistics pk, val, dasc, and ddes.

Theorem 4. $P^{(\mathrm{pk}, \mathrm{val}, \mathrm{dasc}, \mathrm{ddes})}(s, t, u, v ; x)=\frac{t \frac{\partial}{\partial x} V(s t,(u+v) / 2 ; x)}{1-s t V(s t,(u+v) / 2 ; x)}$

Proof. First, observe that $\operatorname{val}(\pi)=\mathrm{pk}(\pi)+1$ for all permutations $\pi$, and that the reversal $r(\pi):=\pi_{n} \pi_{n-1} \cdots \pi_{1}$ of a permutation is a (pk, val)-preserving involution on $\mathfrak{S}_{n}$ that 
switches double ascents with double descents. Thus, we have

$$
P_{n}^{(\mathrm{pk}, \mathrm{val}, \mathrm{dasc}, \mathrm{ddes})}(s, t, u, v)=t P_{n}^{(\mathrm{pk}, \mathrm{dbl})}\left(s t, \frac{1}{2}(u+v)\right)
$$

for all $n \geqslant 1$, which proves the result in light of Theorem 3 .

Theorem 4 and the formula (4) for $V(s, t ; x)$ can be used together to derive the closed-form formula

$$
P^{(\mathrm{pk}, \mathrm{val}, \text { dasc, ddes })}(s, t, u, v ; x)=\frac{2 t}{\alpha \operatorname{coth}\left(\frac{1}{2} \alpha x\right)-u-v}
$$

where $\alpha=\sqrt{(u+v)^{2}-4 s t}$. This is equivalent to a classical formula of Carlitz and Scoville [5]; see also [19, Exercise 1.61a].

The following corollary states several specializations of Theorem 4 .

\section{Corollary 5.}

(a) $A(t ; x)=\frac{\frac{\partial}{\partial x} V(t,(1+t) / 2 ; x)}{1-t V(t,(1+t) / 2 ; x)}$

(b) $P^{\mathrm{pk}}(t ; x)=\frac{\frac{\partial}{\partial x} V(t, 1 ; x)}{1-t V(t, 1 ; x)}$

(c) $P^{\text {ddes }}(t ; x)=\frac{\frac{\partial}{\partial x} V(1,(1+t) / 2 ; x)}{1-V(1,(1+t) / 2 ; x)}$

Further specializing Corollary 5 (a) at $t=-1$ implies Theorem 1 (a).

Next we show that the $(-1)$-evaluation of the double descent distribution over $\mathfrak{S}_{2 n+1}$ gives the tangent number $E_{2 n+1}$.

Theorem 6. For all $n \geqslant 1$, we have

$$
P_{n}^{\mathrm{ddes}}(-1)= \begin{cases}E_{n}, & \text { if } n \text { is odd, } \\ 0, & \text { if } n \text { is even } .\end{cases}
$$

Thus $P^{\mathrm{ddes}}(-1 ; x)=\tan (x)$.

Proof. Comparing Theorem 3 with Corollary 5 (c), we have $P^{\text {ddes }}(-1 ; x)=P^{\text {(pk,dbl) }}(1,0 ; x)$, which implies $P_{n}^{\text {ddes }}(-1)=P_{n}^{(\mathrm{pk}, \mathrm{dbl})}(1,0)$ for all $n \geqslant 1$. Observe that $P_{n}^{(\mathrm{pk}, \mathrm{dbl})}(1,0)$ is the number of permutations in $\mathfrak{S}_{n}$ with no double ascents or double descents. It is easy to see that there are no such permutations for even $n$, and the only such permutations for odd $n$ are alternating permutations: permutations $\pi=\pi_{1} \pi_{2} \cdots \pi_{n}$ satisfying $\pi_{1}<\pi_{2}>\pi_{3}<$ $\pi_{4}>\cdots<\pi_{n}$. It is well known that there are $E_{n}$ alternating permutations in $\mathfrak{S}_{n}$, and the proof follows.

Similar reasoning can be used to prove the formula (1) for Eulerian polynomials evaluated at $t=-1$. 


\section{Counting derangements by cyclic statistics}

Recall that a derangement is a permutation with no fixed points, i.e., a permutation for which $\pi_{i} \neq i$ for all $i$. Let $\mathfrak{D}_{n}$ be the set of derangements in $\mathfrak{S}_{n}$. Our goal in this section is to provide an analogous treatment of the material from the previous section but for counting derangements with respect to several "cyclic statistics" that we will define shortly.

When writing permutations in cycle notation, we adopt the convention of writing each cycle with its largest letter in the first position, and writing the cycles from left-to-right in increasing order of their largest letters. (This convention is sometimes called canonical cycle representation.) For example, the permutation $\pi=649237185$ in one-line notation is written as $\pi=(42)(716)(8)(953)$ in cycle notation.

Given $\pi=\pi_{1} \pi_{2} \cdots \pi_{n}$, we say that $\pi_{i}$ is:

- a cyclic peak if $i<\pi_{i}>\pi_{\pi_{i}}$;

- a cyclic valley if $i>\pi_{i}<\pi_{\pi_{i}}$;

- a cyclic double ascent if $i<\pi_{i}<\pi_{\pi_{i}}$;

- a cyclic double descent if $i>\pi_{i}>\pi_{\pi_{i}}$.

Every letter of a derangement is either a cyclic peak, cyclic valley, cyclic double ascent, or cyclic double descent. Define $\operatorname{cpk}(\pi), \operatorname{cval}(\pi), \operatorname{cdasc}(\pi)$, and $\operatorname{cddes}(\pi)$ to be the number of cyclic peaks, cyclic valleys, cyclic double ascents, and cyclic double descents of $\pi$, respectively.

These "cyclic statistics" were studied earlier by, e.g., Zeng [23], Shin and Zeng [17], and Sun and Wang [21]. ${ }^{6}$ These statistics are also closely related to a classical permutation statistic, the excedance number. We say that $i \in[n]$ is an excedance of $\pi$ if $i<\pi_{i}$ and let $\operatorname{exc}(\pi)$ denote the number of excedances of $\pi$. Then $i$ is an excedance of $\pi$ if and only if $i$ is a cyclic valley or cyclic double ascent of $\pi$, and it is well-known that the excedance number exc and the descent number des are equidistributed over $\mathfrak{S}_{n}$.

Define the map $o: \mathfrak{S}_{n} \rightarrow \mathfrak{S}_{n}$, where the input is a permutation in canonical cycle representation and the output is a permutation in one-line notation, by erasing the parentheses. Continuing the example with $\pi=(42)(716)(8)(953)$, we have $o(\pi)=$ 427168953. The map $o$ is often called Foata's transformation fondamentale and first appeared in [8] (see also [9]). It is easy to see that the transformation fondamentale is a bijection; we can recover the cycles of $\pi$ by noting the left-to-right maxima of $o(\pi)$ : given a permutation $\sigma=\sigma_{1} \sigma_{2} \cdots \sigma_{n}$, we say that $\sigma_{i}$ is a left-to-right maximum of $\sigma$ if $\sigma_{j}<\sigma_{i}$ for all $1 \leqslant j<i$.

Our work in this section will rely on a cyclic variant of valley-hopping introduced in [21]. Define $\theta_{k}: \mathfrak{D}_{n} \rightarrow \mathfrak{D}_{n}$ by $\theta_{k}(\pi)=o^{-1}\left(\varphi_{k}(o(\pi))\right)$, where the 0th letter of $o(\pi)$ is treated as 0 rather than $\infty$. Similarly, for a subset $S \subseteq[n]$, define $\theta_{S}: \mathfrak{D}_{n} \rightarrow \mathfrak{D}_{n}$ by $\theta_{S}=\prod_{k \in S} \theta_{k}$.

\footnotetext{
${ }^{6}$ Chow et al. [6] also derived various formulas for counting permutations by cyclic peaks and cyclic valleys, but their definitions for these statistics differ from ours in that they do not allow the first or last letter of a cycle to be a cyclic peak or cyclic valley.
} 
Then the cyclic modified Foata-Strehl action (or cyclic valley-hopping) is the $\mathbb{Z}_{2}^{n}$-action defined by the involutions $\theta_{S}$. It is easy to see that cyclic valley-hopping toggles between cyclic double ascents and cyclic double descents, but does not change cyclic peaks or cyclic valleys.

Let $\widetilde{\mathfrak{D}}_{n}$ denote the set of derangements of $[n]$ with no cyclic double ascents and where each cyclic double descent is assigned one of two colors: red or blue. Then cyclic valleyhopping induces a map $\stackrel{\circ}{\Phi}$ from $\widetilde{\mathfrak{D}}_{n}$ to $\mathfrak{D}_{n}$ defined in the analogous way as the map $\Phi$ from Section 2 , but with $R(\pi)$ being the set of red cyclic double descents. It then follows from the same reasoning as in the proof of Lemma 2 that $\stackrel{\circ}{\Phi}$ is a (cpk, cdbl)-preserving bijection, where $\operatorname{cdbl}(\pi):=\operatorname{cdasc}(\pi)+\operatorname{cddes}(\pi)$ is the total number of cyclic double ascents and cyclic double descents of $\pi$.

\subsection{A cyclic analogue of Theorem 3 for derangements}

For permutation statistics st $\mathrm{st}_{1}, \mathrm{st}_{2}, \ldots, \mathrm{st}_{m}$ and variables $t_{1}, t_{2}, \ldots, t_{m}$, we define the polynomials $\left\{D_{n}^{\left(\mathbf{s t}_{1}, \mathbf{s t}_{2}, \ldots \mathbf{s t}_{m}\right)}\left(t_{1}, t_{2}, \ldots, t_{m}\right)\right\}_{n \geqslant 0}$ by

$$
D_{n}^{\left(\mathbf{s t}_{1}, \mathbf{s t}_{2}, \ldots \mathbf{s t}_{m}\right)}\left(t_{1}, t_{2}, \ldots, t_{m}\right):=\sum_{\pi \in \mathfrak{D}_{n}} t_{1}^{\mathrm{st}_{1}(\pi)} t_{2}^{\mathrm{st}_{2}(\pi)} \cdots t_{m}^{\mathrm{st}_{m}(\pi)}
$$

and we let

$$
D^{\left(\mathbf{s t}_{1}, \mathrm{st}_{2}, \ldots . \mathbf{s t}_{m}\right)}\left(t_{1}, t_{2}, \ldots, t_{m} ; x\right):=1+\sum_{n=1}^{\infty} D_{n}^{\left(\mathrm{st}_{1}, \mathrm{st}_{2}, \ldots \mathbf{s t}_{m}\right)}\left(t_{1}, t_{2}, \ldots, t_{m}\right) \frac{x^{n}}{n !}
$$

be their exponential generating function. ${ }^{7}$ These encode the distributions of permutation statistics over derangements.

We now present a cyclic analogue of Theorem 3 for derangements.

Theorem 7. $D^{(\mathrm{cpk}, \mathrm{cdbl})}(s, t ; x)=\frac{1}{1-s V(s, t ; x)}$

Proof. It suffices to show that

$$
D_{n}^{(\mathrm{cpk}, \mathrm{cdbl})}(s, t)=\sum_{k=0}^{\lfloor n / 2\rfloor} s^{k} \sum_{B} U_{\left|B_{1}\right|-2}(s, t) \cdots U_{\left|B_{k}\right|-2}(s, t)
$$

where the second sum is over all ordered set partitions $B$ of $[n]$ into parts $B_{1}, \ldots, B_{k}$. We interpret the right-hand side of (6) as in the proof of Theorem 3 (without the initial block $B_{0}$ with an $\infty$ ) and apply the same sign-reversing involution; the objects that remain after cancellation are of the form

$$
c_{1}>c_{2}>\cdots>c_{\left|B_{0}\right|}\left|c_{\left|B_{0}\right|+1}>c_{\left|B_{0}\right|+2}>\cdots>c_{\left|B_{0}\right|+\left|B_{1}\right|}\right| \cdots \mid c_{n-\left|B_{k}\right|+1}>\cdots>c_{n}
$$

with no dominoes and such that $c_{i}<c_{i+1}$ whenever $c_{i}$ and $c_{i+1}$ are in separate blocks.

\footnotetext{
${ }^{7}$ As before, if we have a single statistic st, we write these simply as $D_{n}^{\text {st }}(t)$ and $D^{\text {st }}(t ; x)$.
} 
Now, rather than treating these remaining objects as permutations in one-line notation, we want to treat them as permutations in cycle notation with blocks corresponding to cycles. In doing so, we merge two adjacent blocks whenever the first element of the second block in the pair is not larger than all elements from all preceding blocks, i.e., whenever the element is not a left-to-right maximum of the underlying permutation written in one-line notation; this guarantees that the resulting permutations are correctly written in canonical cycle representation and is clearly reversible.

Moreover, these permutations are derangements because each block has size at least 2, and they have no cyclic double ascents and have each cyclic double descent colored either red or blue (depending on the color of the corresponding monomino). In other words, these permutations $\pi$ are precisely the elements of $\widetilde{\mathfrak{D}}_{n}$ and each contributes a weight of $s^{\mathrm{cpk}(\pi)} t^{\mathrm{cdbl}(\pi)}$ to the right-hand side of Equation (6). The result then follows from applying the (cpk, cdbl)-preserving bijection $\stackrel{\circ}{\Phi}$.

We extend Theorem 7 to an analogous result for the joint distribution of the statistics cpk, cval, cdasc, and cddes over $\mathfrak{D}_{n}$.

Theorem 8. $D^{(\mathrm{cpk}, \mathrm{cval}, \text { cdasc,cddes })}(s, t, u, v ; x)=\frac{1}{1-s t V(s t,(u+v) / 2 ; x)}$

Proof. First, observe that $\operatorname{cpk}(\pi)=\operatorname{cval}(\pi)$ for all derangements $\pi$. The cyclic valleyhopping map $\theta_{S}$, where $S$ is the set containing all cyclic double ascents and cyclic double descents of $\pi$, is a (cpk, cval)-preserving involution on $\mathfrak{D}_{n}$ that switches cyclic double ascents with cyclic double descents. Thus, we have

$$
D_{n}^{(\mathrm{cpk}, \mathrm{cval}, \mathrm{cdasc}, \mathrm{cddes})}(s, t, u, v)=D_{n}^{(\mathrm{pk}, \mathrm{dbl})}\left(s t, \frac{1}{2}(u+v)\right)
$$

for all $n \geqslant 1$, which along with Theorem 7 proves the result.

We can use Theorem 8 and Equation (4) to derive the formula

$$
D^{(\mathrm{cpk}, \mathrm{cval}, \mathrm{cdasc}, \mathrm{cddes})}(s, t, u, v ; x)=\frac{\alpha e^{-\frac{1}{2}(u+v) x}}{\alpha \cosh \left(\frac{1}{2} \alpha x\right)-(u+v) \sinh \left(\frac{1}{2} \alpha x\right)}
$$

where $\alpha=\sqrt{(u+v)^{2}-4 s t}$. Furthermore, given any permutation $\pi$, let $\operatorname{cyc}(\pi)$ denote the number of cycles of $\pi$ and let fix $(\pi)$ be the number of fixed points of $\pi$. Standard applications of the exponential formula (see [20, Section 5.1]) yield the identities

$$
D^{\text {(cpk,cval,cdasc,cddes,cyc) }}(s, t, u, v, w ; x)=D^{(\mathrm{cpk}, \mathrm{cval}, \text { cdasc,cddes })}(s, t, u, v ; x)^{w}
$$

and

$$
1+P^{(\mathrm{cpk}, \mathrm{cval}, \mathrm{cdasc}, \mathrm{cddes}, \mathrm{cyc}, \mathrm{fix})}(s, t, u, v, w, y ; x)=e^{w y x} D^{(\mathrm{cpk}, \mathrm{cval}, \mathrm{cdasc}, \mathrm{cddes}, \mathrm{cyc})}(s, t, u, v, w ; x) .
$$


Then, combining Equations (7), (8), and (9) yields the following exponential generating function formula for the sextuple distribution (cpk, cval, cdasc, cddes, cyc, fix) over all permutations:

$$
1+P^{(\mathrm{cpk}, \mathrm{cval}, \mathrm{cdasc}, \mathrm{cddes}, \mathrm{cyc}, \mathrm{fix})}(s, t, u, v, w, y ; x)=\left(\frac{\alpha e^{\left(y-\frac{1}{2}(u+v)\right) x}}{\alpha \cosh \left(\frac{1}{2} \alpha x\right)-(u+v) \sinh \left(\frac{1}{2} \alpha x\right)}\right)^{w} .
$$

An equivalent form of this formula was proven earlier by Zeng [23, Théorème 1].

\subsection{Counting derangements by excedances}

In the remainder of this section, we examine specializations of Theorem 8 that give rise to formulas for individual cyclic statistics, beginning with the excedance number.

The excedance polynomials $D_{n}(t):=D_{n}^{\text {exc }}(t)$ have been well-studied; for example, it is known that they have exponential generating function

$$
D(t ; x):=D^{\mathrm{exc}}(t ; x)=\frac{(1-t) e^{-x}}{e^{-(1-t) x}-t}
$$

[14] and are $\gamma$-positive $[2,17,21]$. From Theorem 8 we obtain the following.

Corollary 9. $D(t ; x)=\frac{1}{1-t V(t,(1+t) / 2 ; x)}$

It follows from Corollary 9 that the exponential generating function for the excedance polynomials $D(t ; x)$ evaluated at $t=-1$ is the reciprocal of the exponential generating function $F(x)=\cosh (x)$ for the sequence $1,0,1,0, \ldots$

Corollary 10. $D(-1 ; x)=F(x)^{-1}$

This identity can be used to rederive the classical result due to Roselle [14] that

$$
D_{n}(-1)= \begin{cases}(-1)^{n / 2} E_{n}, & \text { if } n \text { is even } \\ 0, & \text { if } n \text { is odd }\end{cases}
$$

We note that, from Equation (2), one can show that

$$
U_{n}(t,(1+t) / 2)=1+t+\cdots+t^{n}
$$

for $n \geqslant 1$. Then, using Equation (10), Corollary 9 can be obtained as a specialization of a formula of Shareshian and Wachs [16, Equation (1.4)] involving Eulerian quasisymmetric functions. 


\subsection{Counting derangements by cyclic peaks}

Next, we examine the distribution of the cyclic peak number over derangements.

Corollary 11. $D^{\mathrm{cpk}}(t ; x)=\frac{1}{1-t V(t, 1 ; x)}$

By specializing (7) appropriately, we derive the formula

$$
D^{\mathrm{cpk}}(t ; x)=\frac{\sqrt{1-t} e^{-x}}{\sqrt{1-t} \cosh (x \sqrt{1-t})-\sinh (x \sqrt{1-t})} .
$$

The first several polynomials $D_{n}^{\mathrm{cpk}}(t)$ are given in the following table.

\begin{tabular}{|c|c|c|c|}
\hline$n$ & $D_{n}^{\mathrm{cpk}}(t)$ & $n$ & $D_{n}^{\mathrm{cpk}}(t)$ \\
\hline 1 & 0 & 5 & $8 t+36 t^{2}$ \\
\hline 2 & $t$ & 6 & $16 t+188 t^{2}+61 t^{3}$ \\
\hline 3 & $2 t$ & 7 & $32 t+864 t^{2}+958 t^{3}$ \\
\hline 4 & $4 t+5 t^{2}$ & 8 & $64 t+3728 t^{2}+9656 t^{3}+1385 t^{4}$ \\
\hline
\end{tabular}

Note that the coefficient of $t$ in $D_{n}^{\text {cpk }}(t)$ seems to be $2^{n-2}$ for all $n \geqslant 2$; this is easy to explain combinatorially.

Proposition 12. For all $n \geqslant 2$, the number of derangements in $\mathfrak{D}_{n}$ with exactly one cyclic peak is $2^{n-2}$.

Proof. It is easy to see that every derangement $\pi$ of $[n]$ with exactly one cyclic peak has exactly one cycle and can be written in the form

$$
\left(c_{1} c_{2} \cdots c_{k} c_{k+1} \cdots c_{n}\right)
$$

where $c_{1}=n$ is the only cyclic peak of $\pi$, the sequence $c_{2} \cdots c_{k}$ is decreasing (with $c_{k}=1$ ), and the sequence $c_{k+1} \cdots c_{n}$ is increasing. Thus, for every letter $i$ between 2 and $n-1$, either $i$ belongs to the decreasing sequence or the increasing sequence, and these $n-2$ choices completely determine the derangement $\pi$.

It follows from Corollary 11 that the exponential generating function for the numbers $D_{n}^{\mathrm{cpk}}(-1)$ is the reciprocal of the exponential generating function $G(x)$ for the Pell numbers.

Corollary 13. $D^{\mathrm{cpk}}(-1 ; x)=G(x)^{-1}$

We do not have a combinatorial interpretation for the numbers $D_{n}^{\text {cpk }}(-1)$ themselves. The first several of these numbers appear in the following table.

\begin{tabular}{c|cccccccccc}
$n$ & 1 & 2 & 3 & 4 & 5 & 6 & 7 & 8 & 9 & 10 \\
\hline$D_{n}^{\mathrm{cpk}}(-1)$ & 0 & -1 & -2 & 1 & 28 & 111 & -126 & -4067 & -26280 & 53663
\end{tabular}




\subsection{Counting derangements by cyclic double descents}

Lastly, we study the distribution of the cyclic double descent number over derangements.

Corollary 14. $D^{\text {cddes }}(t ; x)=\frac{1}{1-V(1,(1+t) / 2 ; x)}$.

The exponential generating function formula

$$
D^{\text {cddes }}(t ; x)=\frac{\beta e^{-\frac{1}{2}(1+t) x}}{\beta \cosh \left(\frac{1}{2} \beta x\right)-(1+t) \sinh \left(\frac{1}{2} \beta x\right)},
$$

where $\beta=\sqrt{(t+3)(t-1)}$, can be obtained by specializing $(7)$. The first several of the polynomials $D_{n}^{\text {cddes }}(t)$ appear in the following table.

\begin{tabular}{c|ccc|c}
$n$ & $D_{n}^{\text {cddes }}(t)$ & & $n$ & $D_{n}^{\text {cddes }}(t)$ \\
\hline 1 & 0 & & 5 & $19+21 t+3 t^{2}+t^{3}$ \\
2 & 1 & & 6 & $109+98 t+53 t^{2}+4 t^{3}+t^{4}$ \\
3 & $1+t$ & & 7 & $588+808 t+334 t^{2}+118 t^{3}+5 t^{4}+t^{5}$ \\
4 & $6+2 t+t^{2}$ & & 8 & $4033+5766 t+3827 t^{2}+952 t^{3}+248 t^{4}+6 t^{5}+t^{6}$
\end{tabular}

An increasing run of a permutation $\pi$ is a maximal increasing consecutive subsequence of $\pi$ (in one-line notation). For example, the increasing runs of $\pi=467192685$ are $467,19,268$, and 5 . We call increasing runs of length 1 short runs. The sequence of constant coefficients of $D_{n}^{\text {cddes }}(t)$ matches the OEIS sequence [18, A097899] for the number of permutations of $[n]$ with no short runs; this can be verified by comparing the evaluation $D^{\text {cddes }}(0 ; x)$ with the exponential generating function of this OEIS entry, but we give a bijective proof below.

For the purpose of this proof, let us temporarily modify our convention for cycle notation so that we write each cycle with its smallest letter in the first position, and write the cycles from left-to-right in decreasing order of their smallest letters. For example, whereas we previously wrote $\pi=649237185$ as $\pi=(42)(716)(8)(953)$ in canonical cycle representation, now we write $\pi$ as $\pi=(8)(395)(24)(167)$. Let $o^{\prime}: \mathfrak{S}_{n} \rightarrow \mathfrak{S}_{n}$ be the map defined by taking a permutation in cycle notation under this new convention and erasing the parentheses, yielding a permutation in one-line notation. This is a bijection; we can recover the cycles of $\pi$ by noting the left-to-right minima of $o(\pi)$ : given a permutation $\sigma=\sigma_{1} \sigma_{2} \cdots \sigma_{n}$, we say that $\sigma_{i}$ is a left-to-right minimum of $\sigma$ if $\sigma_{j}>\sigma_{i}$ for all $1 \leqslant j<i$.

Proposition 15. An letter $i \in[n]$ is a fixed point or cyclic double descent of $\pi \in \mathfrak{S}_{n}$ if and only if $i$ is a short run of $o^{\prime}(\pi)$.

In particular, this proposition implies that the number of derangements of $[n]$ with no cyclic double descents is equal to the number of permutations of $[n]$ with no short runs. 
Proof. Fix a permutation $\pi \in \mathfrak{S}_{n}$ and a letter $i \in[n]$. Let us write $o^{\prime}(\pi)=\sigma_{1} \sigma_{2} \cdots \sigma_{n}$ in one-line notation, and take $\sigma_{j}=i$. By taking as convention $\sigma_{0}=\infty$ and $\sigma_{n+1}=0$, it is easy to see that $\sigma_{j}=i$ is a short run of $o^{\prime}(\pi)$ if and only if $\sigma_{j-1}>\sigma_{j}>\sigma_{j+1}$.

We divide into cases. First, suppose that $i \in[n]$ is a fixed point of $\pi$. Then $\sigma_{j}$ and $\sigma_{j+1}$ are both left-to-right minima, so $\sigma_{j-1}>\sigma_{j}>\sigma_{j+1}$. Now suppose that $i \in[n]$ is a cyclic double descent of $\pi$. Note that the first letter of a cycle (under our current convention) cannot be a cyclic double descent. If $i$ is neither the first nor last letter of its cycle in $\pi$, then $\sigma_{j-1}>\sigma_{j}>\sigma_{j+1}$. Otherwise, if $i$ is the last letter of its cycle in $\pi$, then $\sigma_{j-1}>\sigma_{j}$ and $\sigma_{j+1}$ is a left-to-right minimum; thus $\sigma_{j-1}>\sigma_{j}>\sigma_{j+1}$. In each case, it follows that $\sigma_{j}=i$ is a short run of $o^{\prime}(\pi)$. Hence, every fixed point and cyclic double descent of $\pi$ is a short run of $o^{\prime}(\pi)$; the reverse direction is similar.

Finally, we give a cyclic analogue of Theorem 6 for derangements.

Theorem 16. For all $n \geqslant 1$, we have

$$
D_{n}^{\text {cddes }}(-1)= \begin{cases}E_{n}, & \text { if } n \text { is even } \\ 0, & \text { if } n \text { is odd } .\end{cases}
$$

Thus $D^{\text {cddes }}(-1 ; x)=\sec (x)$.

In other words, the $(-1)$-evaluation of the cyclic double descent distribution over $\mathfrak{D}_{2 n}$ gives the secant number $E_{2 n}$.

Proof. By comparing Theorem 7 with Corollary 14 , we have $D_{n}^{\text {cddes }}(-1)=D_{n}^{\text {(cpk,cdbl })}(1,0)$ for all $n \geqslant 1$. Observe that $D_{n}^{\text {(cpk,cdbl) }}(1,0)$ is the number of derangements of $[n]$ with no cyclic double ascents or cyclic double descents. Because the number of cyclic peaks of any permutation is equal to its number of cyclic valleys, it is evident that there are no such permutations for odd $n$, and it is easy to see that the map $o$ defined earlier is a bijection between such permutations for an even $n$ and reverse-alternating permutations of $[n]$ : permutations $\pi=\pi_{1} \pi_{2} \cdots \pi_{n}$ satisfying $\pi_{1}>\pi_{2}<\pi_{3}>\pi_{4}<\cdots>\pi_{n}$. Since there are $E_{n}$ reverse-alternating permutations in $\mathfrak{S}_{n}$, the proof follows.

\section{Acknowledgements}

We thank an anonymous referee for providing thoughtful comments which improved the presentation of this paper and for pointing us to several key references from the literature on Chebyshev polynomials. We also thank Mohamed Omar and Justin Troyka for helpful discussions relating to this project.

\section{References}

[1] George E. Andrews, Richard Askey, and Ranjan Roy, Special Functions, Encyclopedia of Mathematics and its Applications, vol. 71, Cambridge University Press, Cambridge, 1999. 
[2] Christos A. Athanasiadis and Christina Savvidou, The local h-vector of the cluster subdivision of a simplex, Sém. Lothar. Combin. 66 (2011/12), Art. B66c, 21 pp.

[3] Arthur T. Benjamin and Daniel Walton, Counting on Chebyshev polynomials, Math. Mag. 82 (2009), no. $2,117-126$.

[4] Petter Brändén, Actions on permutations and unimodality of descent polynomials, European J. Combin. 29 (2008), no. 2, 514-531.

[5] L. Carlitz and Richard Scoville, Generalized Eulerian numbers: combinatorial applications, J. Reine Angew. Math. 265 (1974), 110-137.

[6] Chak-On Chow, Shi-Mei Ma, Toufik Mansour, and Mark Shattuck, Counting permutations by cyclic peaks and valleys, Ann. Math. Inform. 43 (2014), 43-54.

[7] F. N. David and D. E. Barton, Combinatorial Chance, Lubrecht \& Cramer Ltd, 1962.

[8] Dominique Foata, Étude algébrique de certains problèmes d'analyse combinatoire et du calcul des probabilités, Publ. Inst. Statist. Univ. Paris 14 (1965), 81-241.

[9] Dominique Foata and Marcel-P. Schützenberger, Théorie géométrique des polynômes eulériens, Lecture Notes in Mathematics, Vol. 138, Springer-Verlag, Berlin-New York, 1970.

[10] Dominique Foata and Volker Strehl, Rearrangements of the symmetric group and enumerative properties of the tangent and secant numbers, Math. Z. 137 (1974), 257-264.

[11] Percy A. MacMahon, Combinatory Analysis, Two volumes (bound as one), Chelsea Publishing Co., New York, 1960. Originally published in two volumes by Cambridge University Press, 1915-1916.

[12] Earl D. Rainville, Special Functions, The Macmillan Co., New York, 1960.

[13] Theodore J. Rivlin, Chebyshev Polynomials: From Approximation Theory to Algebra and Number Theory, 2nd ed., Pure and Applied Mathematics (New York), John Wiley \& Sons, Inc., New York, 1990.

[14] D. P. Roselle, Permutations by number of rises and successions, Proc. Amer. Math. Soc. 19 (1968), $8-16$.

[15] Louis W. Shapiro, Wen-Jin Woan, and Seyoum Getu, Runs, slides, and moments, SIAM J. Alg. Disc. Meth. 4 (1983), no. 4, 459-466.

[16] John Shareshian and Michelle L. Wachs, Eulerian quasisymmetric functions, Adv. Math. 225 (2010), no. $6,2921-2966$.

[17] Heesung Shin and Jiang Zeng, The symmetric and unimodal expansion of Eulerian polynomials via continued fractions, European J. Combin. 33 (2012), no. 2, 111-127.

[18] N. J. A. Sloane, The On-Line Encyclopedia of Integer Sequences. Published electronically at http: //oeis.org, 2018.

[19] Richard P. Stanley, Enumerative Combinatorics, vol. 1, 2nd ed., Cambridge University Press, 2011.

[20] _ Enumerative Combinatorics, vol. 2, Cambridge University Press, 2001.

[21] Hua Sun and Yi Wang, A group action on derangements, Electron. J. Combin. 21 (2014), no. 1, \#P1.67.

[22] Justin Troyka, Period mimicry: A note on the (-1)-evaluation of the peak polynomials, 2019. arXiv: 1907.06681.

[23] Jiang Zeng, Énumérations de permutations et J-fractions continues, European J. Combin. 14 (1993), no. 4, 373-382.

[24] Yan Zhuang, Counting permutations by runs, J. Comb. Theory Ser. A 142 (2016), 147-176. 